\title{
Effect of Eight Weeks of Aerobic Training on Some Myocardial Fibrosis Indices in Cardiac Muscle of Diabetic Rats
}

\author{
${ }^{1}$ Abolfazl Samiei, ${ }^{1}$ Naser Behpour ${ }^{*},{ }^{1}$ Vahid Tadibi, ${ }^{2}$ Rozita Fathi \\ ${ }^{1}$ Faculty of Physical Education and Sport Sciences, Razi University, Kermanshah, Iran. ${ }^{2}$ Faculty of Physical Education \\ and Sport Sciences, University of Mazandaran, Babolsar, Iran.
}

\begin{abstract}
Background. Myocardial fibrosis is identified as a major side effect of Diabetes Mellitus on the heart. Some biomarkers including the ratio of matrix metalloproteinases and their inhibitors in collagen synthesis and collagen degradation are clinically useful in the diagnosis and identification of myocardial fibrosis. In addition, regular aerobic exercise training is one of the major and non-pharmacological solutions for preserving and promoting cardiovascular health. Objectives. Thus, the current research aims at investigating the effect of aerobic training on levels of matrix metalloproteinase-9 (MMP-9) and tissue inhibitor of matrix metalloproteinase 1 (TIMP1) in diabetic rats. Methods. 30 male Wistar rats were randomly divided into 3 groups (healthy control, diabetic control, and exercise diabetic). Diabetes was induced by intraperitoneal injection of streptozotocin at $55 \mathrm{mg} / \mathrm{kg}$ per body weight. The exercise program comprised eight weeks of running on a bar for 60 minutes at a speed of $25 \mathrm{~m} / \mathrm{min}$. Biopsy was carried out $48 \mathrm{~h}$ after the last exercise session, and cardiac levels of MMP-9 and TIMP-1 values were measured by the ELISA sandwich method. Results. The findings of the current research indicated that diabetes induction caused significant increase in cardiac levels of MMP-9 and significant reduction of TIMP-1 compared to the healthy group. In addition, it was revealed that the implementation of 8 weeks-aerobic exercise significantly reduced cardiac levels of MMP-9 and significantly increased TIMP-1 compared to the group without exercise. Conclusions. The execution of aerobic training in diabetic rats inhibited progressive factors of myocardial fibrosis and thus fibrosis risk in diabetic heart is highly reduced.
\end{abstract}

KEY WORDS: Matrix Metalloproteinase, Tissue Inhibitor of Matrix Metalloproteinase, Aerobic Training, Diabetes.

\section{INTRODUCTION}

Diabetes mellitus is a prevalent and extensive metabolic disorder that is associated with inadequate secretion of insulin or its dysfunction and increased blood sugar (1). In contrast to the benefits of multiple treatment interventions such as diet, regular physical activity, weight control, and commonly used medication treatments, epidemiological studies have revealed a growing trend in complications in these patients largely due to the complex nature of the disease and inadequate patient-adherence to treatment regimes. The main cause of premature death in people with diabetes is cardiovascular diseases (2).

Matrix metalloproteinases (MMPs), also known as matrixin, are a family of calcium and zinc-

*. Corresponding Author:

Naser Behpour

E-mail: n_behpoor@yahoo.com 
dependent neutral endopeptidases that play a key role in regulating cell-matrix composition $(3,4)$. Among matrix metalloproteinases, MMP-2 and MMP-9 have the highest dissemination and activity (5). Studies have shown that decomposition of extracellular matrix components is vital in biological processes. In other words, MMPs activity is necessary and important for extracellular matrix protein decomposition under specific physiologic conditions such as embryo development, angiogenesis, and wound healing. However, their activity is transitory and temporary and are locally controlled by endogenous inhibitors. However, in pathological processes such as diseases and microbial and chemical pathogens, the inflammatory response begins with the activation of histamines and ultimately results in the activation of MMPs (6-10). MMP activity can be completely controlled by tissue inhibitor of matrix metalloproteinase (TIMPs) which are expressed by different types of cells. Overall, according to some researchers, all TIMPs can inhibit all MMPs, the most frequent of which is type 1 (TIMP-1) which is highly similar to other isoforms $(11,12)$. MMP-9 is generated by neutrophils, macrophages, osteoclasts, $\mathrm{T}$ lymphocytes and cancer cells which can affect membrane cell collagen, gelatin, fibronectin, elastin, glycosaminoglycans, proteoglycans and laminin. Given high sugar levels in diabetic patients, metalloproteinase levels are therefore much higher than in healthy individuals and their inhibitor levels are lower (13). The relationship between coronary vessels and high MMP is well recognized, while low MMP levels are associated with inhibition and reduction of cardiovascular diseases (14). Researchers have reported that the molecular basis for ventricular re-formulation in diabetic patients is the MMP/TIMP system, which leads to apoptosis of myocardial cells and fibrosis (15).

Experts and researchers have recommended exercise as one of the main and nonpharmacological solution for cardiovascular health preservation and promotion (16). The relationship between regular physical activity and myocardial fibrosis has not been clearly identified(17, 18), therefore, controversial findings exists to this regard. Xiao et al. (2015) reported that regular physical exercises reduces myocardial fibrosis through the reduction of oxidative factors (19). In addition, Kwak et al. (2011) stated that physical exercises reduce fibrosis and matrix metalloproteinase through AMPK activation (20). However, Russo et al. (2011) and La Gerche et al. (2010) reported that regular aerobic exercise could increase myocardial fibrosis $(21,22)$. On the other hand, few studies have investigated these two factors among diabetic patients and the impact of exercise on them. Farzanegi et al. (2013) reported that after 8 weeks of training, plasma levels of MMP-2 and MMP-9 decreased and TIMP-1 increased in type 2 diabetic patients (23). Furthermore, circulating and plasma levels of MMP-9 and TIMP-1 were not sufficiently accurate to indicate myocardial fibrosis as this occurs in various organs of the body such as the lung, kidneys, and peripheral arteries. The explanation of the effects of regular exercises, especially aerobic exercises on diabetic complications specifically myocardial fibrosis is highly ambiguous. Therefore, the current research aimed at creating an underlying knowledge on exercises and myocardial fibrosis and investigated the effect of eight-week aerobic exercises on MMP-9/TIMP-1 ratio in diabetic rats.

\section{MATERIALS AND METHODS}

Animals. The present research was approved by the ethics committee of Razi University, Kermanshah. The subjects in this study were randomly selected male Wistar rats $(n=30)$ from the Pasteur Institute of Iran (6 weeks of age) with an average weight of $140 \pm 20 \mathrm{~g}$. The mice were transferred to the Animal Laboratory of Mazandaran - Babolsar University under controlled conditions of $12: 12 \mathrm{~h}$ light-dark in groups of 5 rats in transparent polycarbonate shelves at $20-24^{\circ} \mathrm{C}$ temperature and $45-55 \%$ humidity. The animals were fed with food produced by Behparvar Co- Iran ad libitum. In addition, the water needed by the animals were made freely available through specified water bottles. The rats were randomly divided into three groups: healthy control group $(\mathrm{n}=10)$, diabetic control group $(\mathrm{n}=10)$, and exercise diabetic group $(n=10)$ following two weeks after familiarization with the laboratory environment. Diabetes was induced by intraperitoneal injection of $1 \mathrm{~mol} / \mathrm{L}$ streptozotocin (STZ) solution (Sigma Aldrich Co., Germany) at $55 \mathrm{mg} / \mathrm{kg}$ per body weight in citrate buffer $(\mathrm{PH}=5.4)$. Blood glucose 
concentration was measured by glucometer 14 days after injection using blood samples collected from the tail of rats. Blood glucose levels of above $250 \mathrm{mg} / \mathrm{dl}$ was the criterion for diabetes. The control group was injected intraperitoneally to equalize the $0.1 \mathrm{mM}$ citrate buffer injection of the same volume (13).

Training Protocol. Prior to the training protocol implementation, the animals were familiarized with 5 sessions of walking and running on a treadmill at $5-8 \mathrm{~m} / \mathrm{min}$ speed and $0 \%$ inclination for 5-10 min for two weeks. The exercise program was implemented as running on a treadmill without rodent inclination for $60 \mathrm{~min}$ at $25 \mathrm{~m} / \mathrm{min}$ speed (24). The program was executed for 8 weeks at 5 sessions per week. For warming up, the subjects ran for $3 \mathrm{~min}$ at $7 \mathrm{~m} / \mathrm{min}$ speed at the beginning of each session, and then the speed was increased by $2 \mathrm{~m} / \mathrm{min}$ in order to reach the respective speed. In order to cool the body at the end of each exercise session, the speed of the treadmill was reduced inversely to reach the initial speed. The entire exercise program was conducted on a treadmill without slopes in Mazandaran-Iran University.

Biopsy and Measurement. 48 hours after the last exercise session, ketamin and xylazine solution was intraperitoneally injected in the rats and then the heart tissue was collected from their split chest. In order to measure MMP-9 and TIMP-1 levels, ELISA Kit (Eastbiopharm Co, China) was used. All the experiments were carried out at the Biochemistry Laboratory, School of Physical Education and Sports Science, Mazandaran-Babolsar University.

Statistical analysis. In order to investigate significant changes in different groups, SPSS Version 21 and Shapiro-Wilk test were used for data analyses. Considering normal distribution of data, one-way ANOVA was used for statistical analysis. After determining significant differences in the groups, Tukey's post hoc test was used to determine the difference between the groups and to examine the differences between research indexes. Repeated measures ANOVA was used to compare glucose levels in the three groups before and after streptozotocin injection and during slaughter. In this study, $\mathrm{p}<0.05$ denoted significant difference.

\section{RESULTS}

Figure 1 shows the fasting glucose values in the three phases of the study: before streptozotocin injection, two weeks after streptozotocin injection, and during. There was no significant difference in fasting glucose levels among the groups before streptozotocin injection and placebo injection. Following streptozotocin injection, it was observed that fasting blood glucose significantly increased in the groups $(\mathrm{p}<0.001)$, while no change was observed in fasting glucose level in the control group ( $p>0.05)$. In addition, the results showed that implementation of exercises significantly reduced fasting blood glucose level $(\mathrm{p}<0.05)$ in the exercise diabetic group and diabetic control group $(\mathrm{p}<0.05)$.

Figure 2 shows that cardiac levels of MMP-9 significantly increased in the diabetic control group compared to the healthy control group $(\mathrm{p}=0.002)$. Furthermore, a comparison between exercise diabetic group and diabetic control group showed that 8 weeks of exercise caused a reduction in cardiac levels of MMP-9 compared to non-exercise group ( $\mathrm{p}=0.002$ ).

Additionally, the research findings showed (Figure 3) that TIMP-1 was significantly reduced in heart tissue of diabetic control group than in the healthy control group $(\mathrm{p}=0.016)$, and TIMP-1 cardiac levels was significantly increased in exercise diabetic group than in the diabetic control group $(\mathrm{p}=0.001)$.

MMP-9/TIMP-1 ratio for each group is shown in Figure 4. The results specified that MMP9/TIMP-1 ratio was significantly increased in diabetic control group compared to healthy control group $(\mathrm{p}=0.001)$. Moreover, significant reduction was observed in MMP-9/TIMP-1 ratio in exercise diabetic group compared to diabetic control group $(\mathrm{p}<0.001)$.

\section{DISCUSSION}

The present study was aimed at investigating the effect of 8 weeks of aerobic exercise that is, running on treadmill, on some muscular fibrosis indexes including MMP-9 and TIMP-1 in heart tissue of diabetic rats. The results of the study showed that diabetes induction caused $34.7 \%$ increase in MMP-9 level and 23.9\% reduction in TIMP-1 levels, and consequently, a $80.2 \%$ increase in MMP-9/TIMP-1 ratio in the heart tissue of diabetic group compared to the healthy group. High blood sugar may lead to changes in extracellular matrix causing increased inflammation and fibrosis (25). 


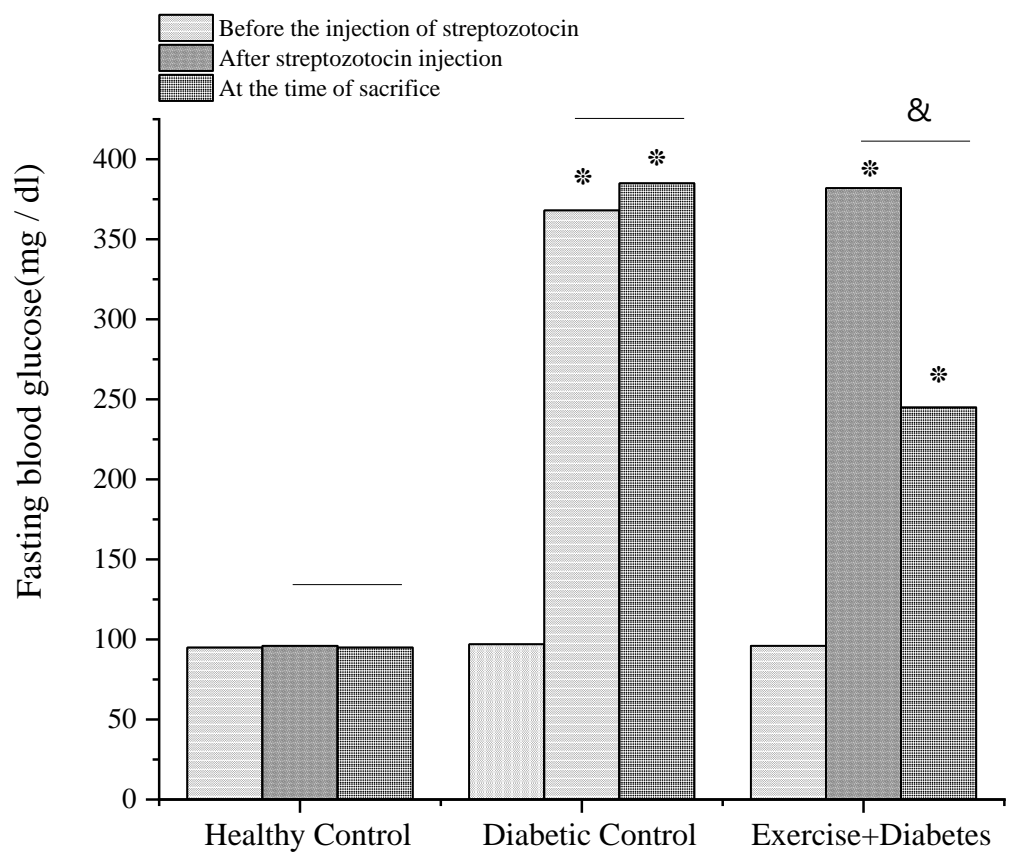

Figure 1. Changes in fasting blood glucose $(\mathrm{mg} / \mathrm{dl})$. *denotes significant differences compared to the stage before streptozotocin injection. \&sign implies significant difference between the stage after streptozotocin injection and the stage after exercises.

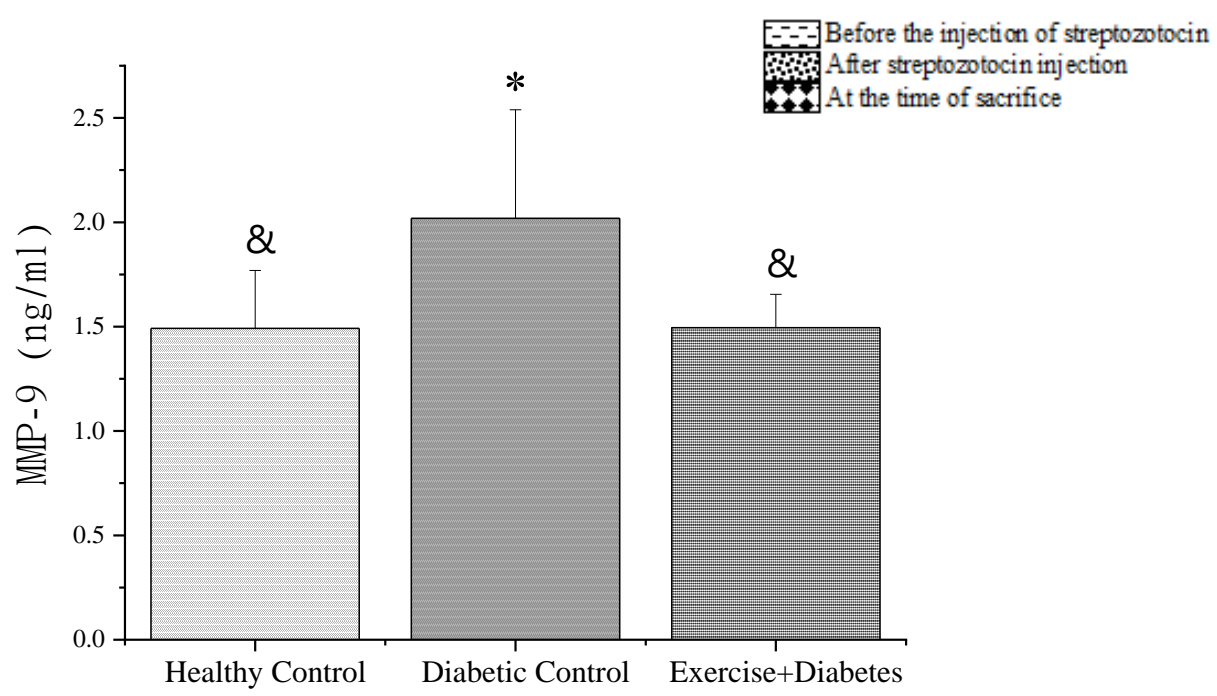

Figure 2. MMP-9 levels in heart tissue. *: Significant difference versus control. \&: Significant difference versus diabetic control. Data are shown based on mean and standard deviation, and significance level is considered at $\mathrm{p}<0.05$. 


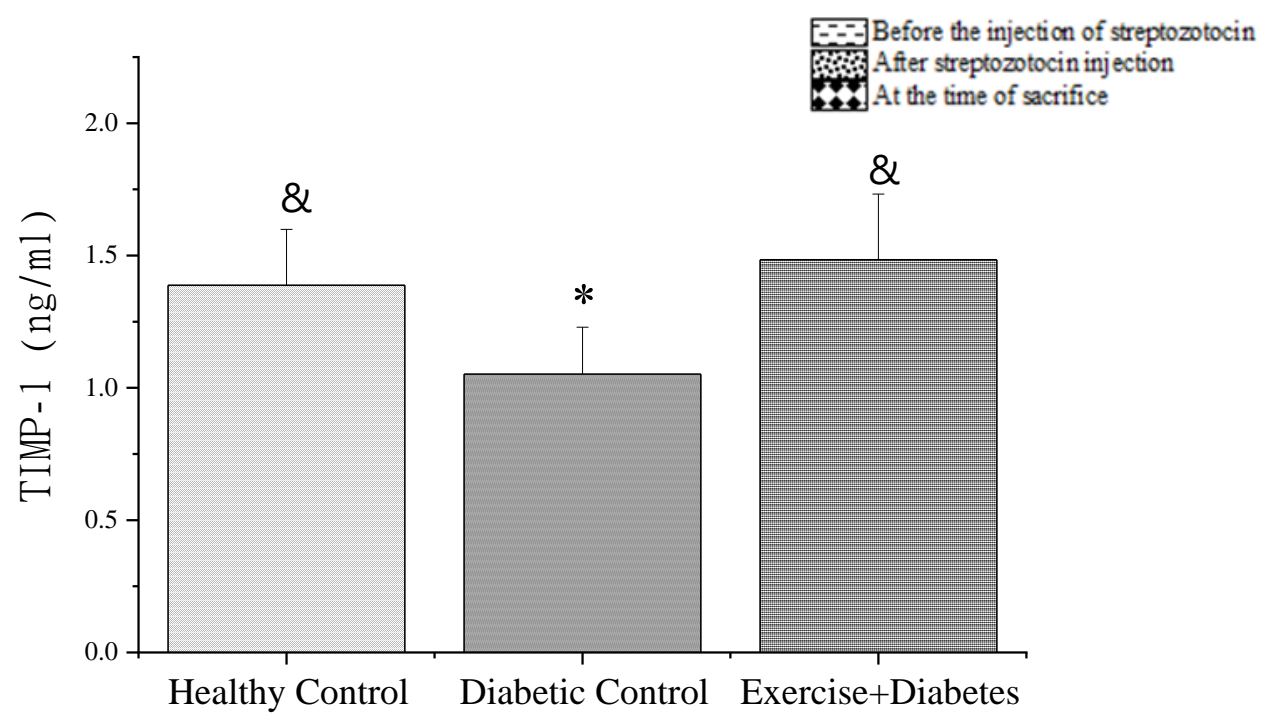

Figure 3. TIMP-1 levels. *: Significant difference versus control. \&: Significant difference versus diabetic control. Data are shown based on mean and standard deviation, and significance level is considered at $\mathrm{p}<0.05$.

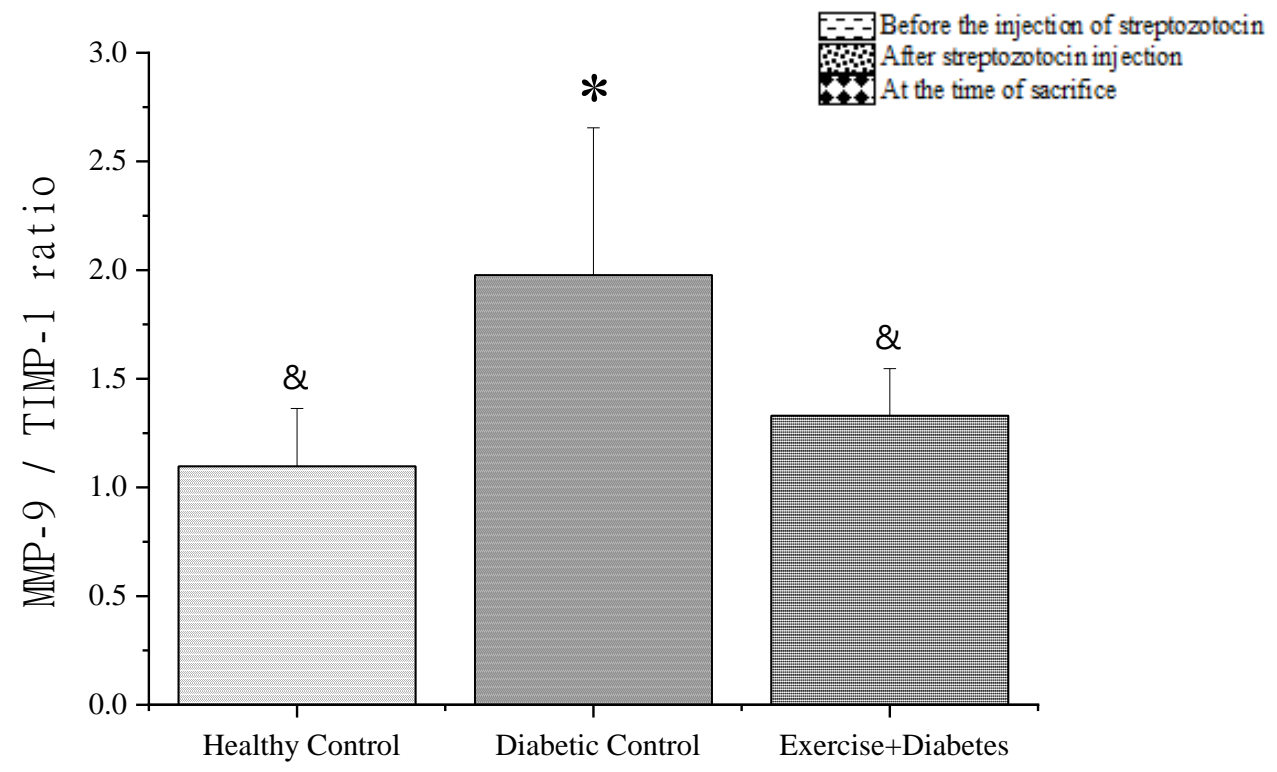

Figure 4. MMP-9/TIMP-1 Ratio. *: Significant difference versus control. \&: Significant difference versus diabetic control. Data are shown based on mean and standard deviation, and significance level is considered at $\mathrm{p}<0.05$.

The results of this study also showed that fasting glucose level in diabetic groups was significantly increased compared to non-diabetic group. Due to high blood glucose levels in 
diabetics, MMPs levels are higher than in healthy subjects, which play a major role in the movement of inflammatory cells, the formation of inflammatory substances, as well as the formation of intercellular matrix structures. Their impairment results in different anomalies in matrix function and ultimately results in inappropriate response to physiological or pathologic changes (26). In addition, Rodriguez et al. (2008) reported that high levels of MMP-9 are directly related to increased risk factors for heart disease in patients with type 2 diabetes and myocardial infarction (27). Consistent with the current research findings, increasing myocardial fibrosis indexes in diabetics have also been confirmed in other researches. Westermann et al. (2007) showed that increased diastolic hardness in diabetic rats is associated with increased cardiac fibrosis (28). Nakamura et al. (2003) reported that MMP-9 and TIMP-1 levels are higher in healthy individuals compared to diabetic individuals (29) and this is in agreement with the current study. In a study by Haffner et al. (2002), it was reported that hyperglycemia treatment reduced serum level of MMP-9 and its other generation stimuli (30). Abnormal ventricular filling has been reported to be associated with high collagen levels in myocardial tissue. Therefore, due to collagen nondegradation in diabetes, the extracellular space is enlarged and reduces the ventricular capacity to accept blood (31). The motility of the ventricular rearrangement in diabetic patients affects MMP/TIMP system which is affected by high glucose levels and leads to apoptosis of the heart muscle cells and subsequently, fibrosis (15). Subsequently, the results of this study showed that 8 weeks of aerobic exercise significantly reduced cardiac levels of MMP-9 by $25.9 \%$, significantly increased MMP-9 levels by $40.9 \%$ and caused significant reduction in MMP-9/TIMP-1 ratio by $47.7 \%$ in diabetic rats. In agreement with the current findings, Kadoglou et al. (2010) reported that 16 weeks of self-control (without trainer) aerobic exercise lead to reduced levels in MMP-9 and MMP-9/TIMP-1 ratio and increased TIMP-1 level in men and women with type diabetes (32). Furthermore, Niessner et al. (2006) observed a reduction in serum level of MMP-9 after 12 weeks of strength exercise (33). Farzanegi et al. (2013) stated that in type 2 diabetic patients, after 8 weeks of training, plasma levels of MMP-2 and MMP-9 decreased and TIMP-1 also increased after 8 weeks of exercise in people with type 2 diabetes (23).

Growing evidences support the protective role of TIMPs as inhibitors of MMP-2 and MMP-9 against cardiovascular diseases $(34,35)$. TIMP-1 as an inhibitor of a wide range of MMPs are inversely associated with plaque atherosclerosis instability and its poor prognosis of cardiovascular disease (36). On the other hand, MMP-9/TIMP-1 ratio is regarded as an independent predictor of cardiovascular disease severity (37). In the current research, MMP-9/TIMP-1 ratio was reduced and cardiac levels of TIMP-1 increased following 8 weeks of aerobic exercise. Therefore, exercise could significantly improve inflammatory and fibrosis factor levels in heart tissue of diabetic rats. Implementing exercises initiated inflammatory responses in the body's protective system cells from preventing cellular damage through the immune cells. It leads to better functioning of a wide range of mediums and solutes and stimuli factors in the immune system, blood and other body fluids (38). These reactions may lead to reduced production of free radicals and finally reduced inflammation and related factors.

\section{CONCLUSION}

The findings of this study showed that exercise training in diabetic rat caused elevated TIMP-1 and reduced fibrosis progressive factors including MMP-9 and MMP-9/TIMP-1 ratio by enhancing protective systems especially in the heart tissue. Thus, the risk of cardiac fibrosis in diabetic individuals was highly reduced.

There were some limitations in carrying out this research. They included: 1) Very rare similar studies were available. 2) The lipid levels of subjects were not measured. For future studies, it is suggested that these indexes are measured and their relationship with changes in MMP-9 and MMP-9/TIMP-1 levels be investigated.

\section{APPLICABLE REMARKS}

- Regular aerobic exercises reduce cardiac fibrosis resulting from diabetes. Thus, diabetic patients can significantly reduce the risk of cardiac fibrosis and heart failure by moderate aerobic exercise training such as running. 


\section{REFERENCES}

1. Johansen T. Treating Diabetes Melitus Using Insulin Injections Administered With Varying Injection Intervals. Google Patents; 2018.

2. Huynh K, Bernardo BC, McMullen JR, Ritchie RH. Diabetic cardiomyopathy: mechanisms and new treatment strategies targeting antioxidant signaling pathways. Pharmacology \& therapeutics. 2014;142(3):375-415.

3. De Boer MD, Selby A, Atherton P, Smith K, Seynnes OR, Maganaris CN, et al. The temporal responses of protein synthesis, gene expression and cell signalling in human quadriceps muscle and patellar tendon to disuse. The Journal of physiology. 2007;585(1):241-51.

4. Li L, Zhao Q, Kong W. Extracellular matrix remodeling and cardiac fibrosis. Matrix Biology. 2018.

5. Kjaer M. Role of extracellular matrix in adaptation of tendon and skeletal muscle to mechanical loading. Physiological reviews. 2004;84(2):649-98.

6. Lee SW, Song KE, Shin DS, Ahn SM, Ha ES, Kim DJ, et al. Alterations in peripheral blood levels of TIMP-1, MMP-2, and MMP-9 in patients with type-2 diabetes. Diabetes research and clinical practice. 2005;69(2):175-9.

7. Lobmann R, Schultz G, Lehnert H. Proteases and the diabetic foot syndrome: mechanisms and therapeutic implications. Diabetes care. 2005;28(2):461-71.

8. Murphy G, Nagase H. Progress in matrix metalloproteinase research. Molecular aspects of medicine. 2008;29(5):290-308.

9. Symeonidis C, Papakonstantinou E, Galli A, Tsinopoulos I, Mataftsi A, Batzios S, et al. Matrix metalloproteinase (MMP-2,-9) and tissue inhibitor (TIMP-1,-2) activity in tear samples of pediatric type 1 diabetic patients. Graefe's Archive for Clinical and Experimental Ophthalmology. 2013;251(3):741-9.

10. Walraven M, Hinz B. Therapeutic approaches to control tissue repair and fibrosis: Extracellular matrix as a game changer. Matrix Biology. 2018.

11. Adachi T, Weisbrod RM, Pimentel DR, Jia Y, Sharov VS, Schöneich C, et al. S-Glutathiolation by peroxynitrite activates SERCA during arterial relaxation by nitric oxide. Nature medicine. 2004;10(11):1200.

12. Murphy G, Docherty AJ. The matrix metalloproteinases and their inhibitors. American journal of respiratory cell and molecular biology. 1992;7:120-.

13. Uemura S, Matsushita H, Li W, Glassford AJ, Asagami T, Lee K-H, et al. Diabetes mellitus enhances vascular matrix metalloproteinase activity. Circulation Research. 2001;88(12):1291-8.

14. Dastani M, Rashidlamir A, Alizadeh A. Effects of 8 Weeks of Aerobic Exercise on Matrix Metalloproteinase-9 and Tissue Inhibitor Levels in Type II Diabetic Women. Zahedan Journal of Research in Medical Sciences. 2014;16(6):12-5.

15. Ho FM, Liu SH, Lin WW, Liau CS. Opposite effects of high glucose on MMP-2 and TIMP-2 in human endothelial cells. Journal of cellular biochemistry. 2007;101(2):442-50.

16. O'keefe JH, Patil HR, Lavie CJ, Magalski A, Vogel RA, McCullough PA, editors. Potential adverse cardiovascular effects from excessive endurance exercise. Mayo Clinic Proceedings; 2012: Elsevier.

17. Philpott J, Houghton K, Luke A. Physical activity recommendations for children with specific chronic health conditions: Juvenile idiopathic arthritis, hemophilia, asthma and cystic fibrosis. Paediatrics \& child health. 2010;15(4):213-8.

18. Roberts TJ, Burns AT, MacIsaac RJ, MacIsaac AI, Prior DL, La Gerche A. Exercise capacity in diabetes mellitus is predicted by activity status and cardiac size rather than cardiac function: a case control study. Cardiovascular diabetology. 2018;17(1):44.

19. Ma X, Fu Y, Xiao H, Song Y, Chen R, Shen J, et al. Cardiac fibrosis alleviated by exercise training is AMPKdependent. PloS one. 2015;10(6):e0129971.

20. Kwak H-B, Kim J-h, Joshi K, Yeh A, Martinez DA, Lawler JM. Exercise training reduces fibrosis and matrix metalloproteinase dysregulation in the aging rat heart. The FASEB Journal. 2011;25(3):1106-17.

21. Russo AD, Pieroni M, Santangeli P, Bartoletti S, Casella M, Pelargonio G, et al. Concealed cardiomyopathies in competitive athletes with ventricular arrhythmias and an apparently normal heart: role of cardiac electroanatomical mapping and biopsy. Heart rhythm. 2011;8(12):1915-22.

22. La Gerche A, Robberecht C, Kuiperi C, Nuyens D, Willems R, de Ravel T, et al. Lower than expected desmosomal gene mutation prevalence in endurance athletes with complex ventricular arrhythmias of right ventricular origin. Heart. 2010;96(16):1268-74.

23. FARZANEGI P, AKBARI A, AZARBAYJANI MA. Effect of Portulaca oleracea Seeds on the Levels of Matrix Metalloproteinase 2, 9 and Tissue Inhibitor Matrix Metalloproteinase 1 in Patients with Type 2 Diabetes. 2013.

24. Soya H, Okamoto M, Matsui T, Lee M, Inoue K, Nishikawa S, et al. Brain activation via exercise: exercise conditions leading to neuronal activation and hippocampal neurogenesis. J Exerc Nutr Biochem. 2011;15:1-10. 
25. Ko SH, Hong OK, Kim JW, Ahn YB, Song KH, Cha BY, et al. High glucose increases extracellular matrix production in pancreatic stellate cells by activating the renin-angiotensin system. Journal of cellular biochemistry. 2006;98(2):343-55.

26. Kim SH, Hong SB, Suh YJ, Choi YJ, Nam M, Lee HW, et al. Association between nutrient intake and obesity in type 2 diabetic patients from the Korean National Diabetes Program: a cross-sectional study. Journal of Korean medical science. 2012;27(10):1188-95.

27. Dominguez-Rodriguez A, Abreu-Gonzalez P, Garcia-Gonzalez MJ, Kaski JC. High serum matrix metalloproteinase- 9 level predict increased risk of in-hospital cardiac events in patients with type 2 diabetes and ST segment elevation myocardial infarction. Atherosclerosis. 2008;196(1):365-71.

28. Westermann D, Rutschow S, Jäger S, Linderer A, Anker S, Riad A, et al. Contributions of inflammation and cardiac matrix metalloproteinase activity to cardiac failure in diabetic cardiomyopathy. Diabetes. 2007;56(3):641-6.

29. Nakamura T, Matsuda T, Suzuki Y, Ueda Y, Koide H. Effects of low-density lipoprotein apheresis on plasma matrix metalloproteinase-9 and serum tissue inhibitor of metalloproteinase-1 levels in diabetic hemodialysis patients with arteriosclerosis obliterans. Asaio Journal. 2003;49:430-4.

30. Haffner SM, Greenberg AS, Weston WM, Chen H, Williams K, Freed MI. Effect of rosiglitazone treatment on nontraditional markers of cardiovascular disease in patients with type 2 diabetes mellitus. Circulation. 2002;106(6):679-84.

31. Mizushige K, Yao L, Noma T, Kiyomoto H, Yu Y, Hosomi N, et al. Alteration in left ventricular diastolic filling and accumulation of myocardial collagen at insulin-resistant prediabetic stage of a type II diabetic rat model. Circulation. 2000;101(8):899-907.

32. Kadoglou N, Vrabas I, Sailer N, Kapelouzou A, Fotiadis G, Noussios G, et al. Exercise ameliorates serum MMP9 and TIMP-2 levels in patients with type 2 diabetes. Diabetes \& metabolism. 2010;36(2):144-51.

33. Niessner A, Richter B, Penka M, Steiner S, Strasser B, Ziegler S, et al. Endurance training reduces circulating inflammatory markers in persons at risk of coronary events: impact on plaque stabilization? Atherosclerosis. 2006;186(1):160-5.

34. Kadoglou NP, Daskalopoulou SS, Perrea D, Liapis CD. Matrix metalloproteinases and diabetic vascular complications. Angiology. 2005;56(2):173-89.

35. Tayebjee MH, Lip GY, Blann AD, MacFadyen RJ. Effects of age, gender, ethnicity, diurnal variation and exercise on circulating levels of matrix metalloproteinases (MMP)-2 and-9, and their inhibitors, tissue inhibitors of matrix metalloproteinases (TIMP)-1 and-2. Thrombosis research. 2005;115(3):205-10.

36. Kruk M, Kalińczuk Ł, Pręgowski J, Przyłuski J, Janas J, Chmielak Z, et al. Serum tissue inhibitor of metalloproteinases-1 and higher risk features of coronary plaque: a volumetric multivessel intravascular ultrasound study. Atherosclerosis. 2007;194(2):e57-e63.

37. Cheng M, Hashmi S, Mao X, Zeng QT. Relationships of adiponectin and matrix metalloproteinase-9 to tissue inhibitor of metalloproteinase-1 ratio with coronary plaque morphology in patients with acute coronary syndrome. Canadian Journal of Cardiology. 2008;24(5):385-90.

38. Kumar V, Abbas AK, Fausto N, Aster JC. Robbins and Cotran pathologic basis of disease, professional edition ebook: elsevier health sciences; 2014. 